\title{
Fine-tuning of unmanned aerial surveillance for ecological systems
}

\author{
$\underline{\text { P.W.J. Baxter }}^{\text {a,b }}$ and G. Hamilton ${ }^{a, b}$ \\ ${ }^{a}$ Plant Biosecurity Cooperative Research Centre, Bruce, Australian Capital Territory \\ ${ }^{b}$ Earth, Environmental and Biological Sciences School, Queensland University of Technology (QUT), \\ Brisbane, Queensland
}

Email:p3.baxter@qut.edu.au

\begin{abstract}
Much of our understanding and management of ecological processes requires knowledge of the distribution and abundance of species. Reliable abundance or density estimates are essential for managing both threatened and invasive populations, yet are often challenging to obtain. Recent and emerging technological advances, particularly in unmanned aerial vehicles (UAVs), provide exciting opportunities to overcome these challenges in ecological surveillance. UAVs can provide automated, cost-effective surveillance and offer repeat surveys for pest incursions at an invasion front. They can capitalise on manoeuvrability and advanced imagery options to detect species that are cryptic due to behaviour, lifehistory or inaccessible habitat. UAVs may also cause less disturbance, in magnitude and duration, for sensitive fauna than other survey methods such as transect counting by humans or sniffer dogs.

The surveillance approach depends upon the particular ecological context and the objective. For example, animal, plant and microbial target species differ in their movement, spread and observability. Lag-times may exist between a pest species presence at a site and its detectability, prompting a need for repeat surveys. Operationally, however, the frequency and coverage of UAV surveys may be limited by financial and other constraints, leading to errors in estimating species occurrence or density.
\end{abstract}

We use simulation modelling to investigate how movement ecology should influence fine-scale decisions regarding ecological surveillance using UAVs. Movement and dispersal parameter choices allow contrasts between locally mobile but slow-dispersing populations, and species that are locally more static but invasive at the landscape scale.

We find that low and slow UAV flights may offer the best monitoring strategy to predict local population densities in transects, but that the consequent reduction in overall area sampled may sacrifice the ability to reliably predict regional population density. Alternative flight plans may perform better, but this is also dependent on movement ecology and the magnitude of relative detection errors for different flight choices.

Simulated investigations such as this will become increasingly useful to reveal how spatio-temporal extent and resolution of UAV monitoring should be adjusted to reduce observation errors and thus provide better population estimates, maximising the efficacy and efficiency of unmanned aerial surveys.

Keywords: Surveillance, probability of detection, weed, pathogen, mammal 


\section{INTRODUCTION}

Much of our understanding and management of ecological processes require knowledge about the distribution and abundance of species. Reliable estimates of abundance or density can be essential for successful population management, whether for conservation, sustainable harvest or biosecurity. These are often financially and logistically challenging to obtain.

Exciting opportunities to overcome challenges in surveillance and monitoring are emerging, due to rapidly adapting and improving new technology (Kalaris et al. 2014). Fixed and mobile sensors on terrestrial, aquatic or aerial robotic devices can provide remote detection overcoming the traditional obstacles of repeatability, accessibility and range. Unmanned aerial vehicles (UAVs in particular are expected to revolutionise the collection of ecological data (Anderson and Gaston 2013), being highly transportable, affordable and versatile (Campbell et al. 2015). UAVs can exploit a range of imagery options for detection of organisms or their impacts (such as symptomatic manifestations of pathogen infection). With precise navigation and reduction of instrumentation size and weight, UAVs offer much closer, finer and ecologically appropriate spatio-temporal resolution than older remote-sensing instruments which are generally mounted on larger aircraft (Anderson and Gaston 2013). Once legal requirements are met, there appear to be few practical disadvantages to UAVs, apart from a reliance on low-wind conditions especially for take-off and landing (Campbell et al. 2015).

The use of UAVs for monitoring ecological populations involves a pathway of implementation steps-image capture, merging, processing, and analysis for species identification - that can each generate potential errors in true detection. Decisions taken along this pathway (choices regarding camera height and angle, aircraft speed, algorithms for image processing and analysis) can involve trade-offs in the consequent levels of detection error. The management implications of these detection errors require an understanding of the appropriate spatio-temporal scale and resolution of autonomous surveillance (Jurdak et al. 2015).

Previous studies have highlighted ways in which spatiotemporal resolution affects the efficacy and efficiency of more traditional monitoring. For example, trade-offs between sampling intensity at a single location and costs of travel between sites leads to a complex relationship between survey costs and sample size per site (Jorgensen et al. 2003), and thus affects which spatio-temporal monitoring decisions are optimal for an overall area (McDonald-Madden et al. 2008).

Here we present a grid-based model of three idealised organisms with distinct population growth and movement patterns, and investigate how the choices in UAV flight plans may influence their population size estimates. Given the growing use of new technology such as UAVs, and their enormous potential contribution to environmental management, it is timely to investigate whether general rules can be established for UAV sampling strategies, that may reflect the movement patterns of organisms.

\section{SPECIES POPULATION AND MOVEMENT DYNAMICS}

\subsection{Landscape}

We model population density and surveillance over a $50 \times 50$ cell grid. To address boundary effects we also include a 3 -cell outer buffer (producing a $56 \times 56$ grid overall). Individuals occurring in the buffer zone do not experience population growth or directional spread (see below), but can still undergo random movement and therefore may re-enter the grid.

\subsection{Three study species}

We ascribe contrasting movement ecology to three simulated species: a relatively territorial mammal-like organism with zero population growth, a plant with high population growth and directional spread, and a plant disease with slow spread and random "movement" reflecting random spread to infect adjacent plants. We refer to these species loosely as "koala", "weed", and "rust", respectively, noting that our parameters are chosen to emphasise contrasting movement ecologies rather than to represent reality. Efficient monitoring of species such as these is important: there are considerable economic costs associated with management of koalas (Field et al. 2004), weeds (Sinden et al. 2004) and plant rusts (Puccinia spp; Hodson 2011, Carnegie and Cooper 2011). 
We populated the grid with 25 individuals, i.e. an initial population density of $1 \%$. The "koala" individuals were assigned random positions across the grid, whereas the invasive species ("weed" and "rust") were assigned random positions within the three most southerly rows, to represent an incursion event. We assumed that grid cells could accommodate multiple individuals.

\subsection{Population growth and movement}

The population growth, spread and random movement of the three species are detailed in Table 1, and example time-lapse distributions are given in Figure 1. Spread was assumed to occur in a northerly direction, which may be interpreted as influence from an environmental driver, e.g., prevailing wind or rain direction. For the "weed", only new propagules were allowed to spread, mimicking seed dispersal and germination: the destination cell was set to 4 grid cells north of the parent cell, perturbed in both latitudinal and longitudinal directions by \pm 2 cells (i.e., chosen from a $[-2,+2]$ triangular distribution). We also used triangular distributions to select destination cells for the undirected random movement of "koalas" and "rusts".

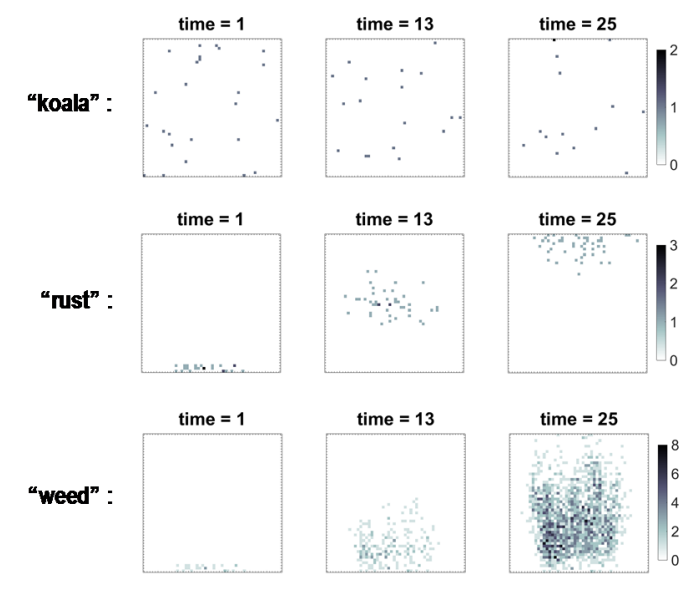

Figure 1. Population densities over time and space.

\section{SPATIAL SAMPLING}

\section{1. $\quad$ Flight-path options}

Table 1. Population and movement traits for the three simulated species.

We assume that UAVs are flown in an east-west direction, thus sampling transects along rows of the grid (a reasonable assumption e.g. for agricultural crops planted in rows). We contrast four different flight options varying by height and speed. We assume that fast flights can sample more of the region (twice the number of rows per sampling period) than slow flights. Similarly, we assume that low-altitude flights sample one row at a time, whereas high altitude flights can sample adjacent rows. The number of rows sampled (in repeating eight-row blocks) is then

$$
N_{8}=(1+F)(1+2 H),
$$

where $F$ and $H$ are indicator variables for fast and high flights, respectively. 


\subsection{Flight-path implications for detection}

The trade-offs in speed and height of image capture have implications for the ability to detect individual organisms, either directly or via their signals, such as pathogenic symptoms produced by rust infections. Slow flights facilitate image processing by allowing more image overlap and better image resolution than fast flights. We therefore assume that slow flights incur less detection error than fast flights. Similarly, we assume that low-altitude flights are prone to less detection error (through higher image resolution and reduced atmospheric attenuation and interference than high altitude flights). We thus calculate probabilities of detecting one individual in each grid-cell given the sampling method for the row sampled (Table 2), scaled to an underlying error rate $\varepsilon$ for convenience. Note that these probabilities are assumed to be independent for each individual, which may not be strictly realistic as individuals could obscure or interfere with each other's

Table 2. Probability of detecting each individual per row (east-west strip) sampled in a repeating eight-row block, using four alternative flight-strategies. Probabilities are expressed in terms of detection errors $(\varepsilon)$; blank entries indicate unsampled rows.

\begin{tabular}{|c|c|c|c|c|}
\hline $\begin{array}{c}\text { Row } \\
\text { number }\end{array}$ & $\begin{array}{c}\text { Low, slow } \\
\left(N_{8}=1\right)\end{array}$ & $\begin{array}{c}\text { Low, fast } \\
\left(N_{8}=2\right)\end{array}$ & $\begin{array}{c}\text { High, slow } \\
\left(N_{8}=3\right)\end{array}$ & $\begin{array}{c}\text { High, fast } \\
\left(N_{8}=6\right)\end{array}$ \\
\hline 1 & & & $1-3 \varepsilon$ & $1-4 \varepsilon$ \\
\hline 2 & $1-\varepsilon$ & $1-2 \varepsilon$ & $1-2 \varepsilon$ & $1-3 \varepsilon$ \\
\hline 3 & & & $1-3 \varepsilon$ & $1-4 \varepsilon$ \\
\hline \multicolumn{5}{|l|}{4} \\
\hline 5 & & & & $1-4 \varepsilon$ \\
\hline 6 & & $1-2 \varepsilon$ & & $1-3 \varepsilon$ \\
\hline 7 & & & & $1-4 \varepsilon$ \\
\hline \multicolumn{5}{|l|}{8} \\
\hline$(9-50)$ & $\begin{array}{c}\text { (sampling } \\
\text { pattern } \\
\text { repeats) }\end{array}$ & $\begin{array}{c}\text { (sampling } \\
\text { pattern } \\
\text { repeats) }\end{array}$ & $\begin{array}{c}\text { (sampling } \\
\text { pattern } \\
\text { repeats) }\end{array}$ & $\begin{array}{c}\text { sampling } \\
\text { pattern } \\
\text { repeats) }\end{array}$ \\
\hline
\end{tabular}
detection signals.

\subsection{Simulation}

We simulated, for 1000 iterations each, the ecological and surveillance processes for the three species and four flight-path options, over 25 time-steps (loosely interpretable as weeks). We repeated the simulation experiment for detection error scales of $\varepsilon=0.05$ and $\varepsilon=0.20$. We calculated the estimated population density, for each row and the overall grid (pro-rata to number of rows sampled), and measured errors in estimating the actual densities as;

$$
\% \text { deviation }=100 \mid \text { actual }- \text { estimated } \mid / \text { actual. }
$$

\section{RESULTS}

\subsection{High detection errors}

At relatively high error levels $(\varepsilon=0.2)$, the ranking of the four flight-paths strategies' performance in estimating row densities was consistent for all three species. Faster flights always produced higher deviation in estimates than slow flights, and for any given speed a high-altitude flight generated higher deviations than those closer to the ground (Figure 2, a-c). For estimating regional (grid-wide) populations, the same rankings held broadly, although some differences emerged for all three species. Low flights were best at predicting "koala" populations but there was little difference between the two flight speeds, and even a slight indication that low, fast flights may be preferable. For "koalas", distributed randomly across the grid (Figure 1), sampling more transects at a per-individual detection probability of 0.6 becomes better than sampling half as many transects at a detection probability of 0.8 per individual. There is some transient sensitivity of population estimates for the two invasive species, as the populations are initially clustered in a few rows of the grid, imparting sampling bias to the schema in Table 2 . 

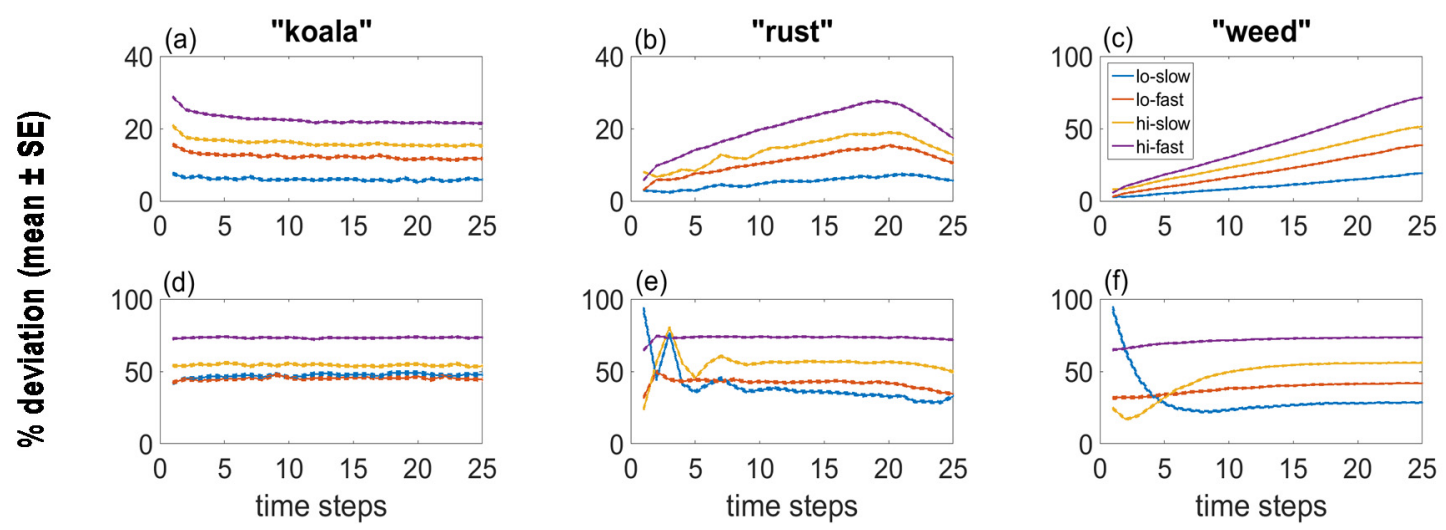

Figure 2. Detection errors in three contrasting simulated species for four flight-sampling modes. Results show deviations in population estimates by row transect (a-c) and overall region (d-f), for 1000 simulations. Detection errors scaled to $\varepsilon=$ 0.20 , so individual detection probabilities range from 0.80 (low-slow flight) to 0.20 (peripheral rows on high-fast flight).

\subsection{Low detection errors}

Increased imaging capability or analytical accuracy, both of which may require additional investment of management resources, can lead to reductions in detection errors. Decreasing our error scale to $\varepsilon=0.05$ reproduces the patterns seen at $\varepsilon=0.2$ in the row-density estimates for all three species; unsurprisingly, the deviations are smaller at the lower error scale (Figure 3, a-c). Interesting differences emerge in the estimates of overall population size (Figure 3, d-f) compared to those based on larger detection errors (Figure 2, d-f). For "koalas", the ranking of flight plan performances for estimating row densities is reversed for estimating population: high and fast flights become preferable. The relative performances of flight-options for the two invasive species are more subtle, although (as with "koalas") the low, slow flight plan is never preferred.

Fast flights generally produce the lowest deviations from actual population size, with "weeds" (which gradually fill up the grid; Figure 1) being better predicted by low, fast flights. In contrast, "rust" (which moves across the grid) is better predicted by high, fast flights. There is again a transient pattern of poor estimation similar to the higher-error scenario, although the performance of high, slow flights are worse.

\section{DISCUSSION AND CONCLUSIONS}
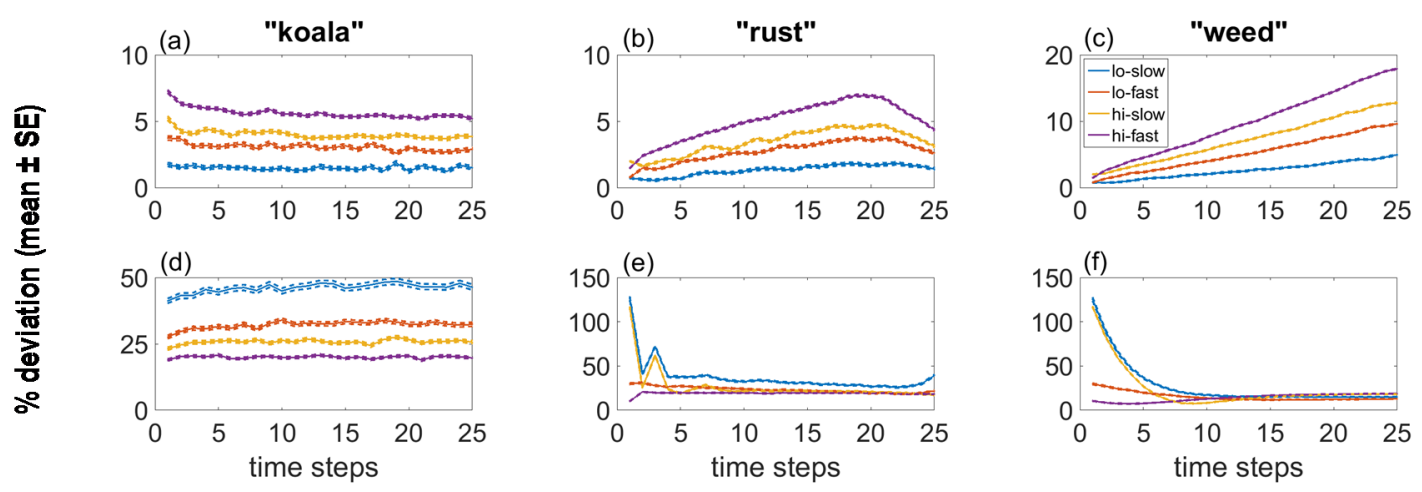

Figure 3. Detection errors in three contrasting simulated species for four flight-sampling modes. Results show deviations in population estimates by row transect (a-c) and overall region (d-f), for 1000 simulations. Detection errors scaled to $\varepsilon=$ 0.05 , so individual detection probabilities range from 0.95 (low-slow flight) to 0.80 (peripheral rows on high-fast flight).

With advances in new technology such as UAVs, ecological monitoring stands on the cusp of a revolution (Anderson and Gaston 2013). It is important therefore to understand how UAV deployment can best serve ecological managers. Our model has shown interesting interactions between detection error levels and spatial and population dynamics of individual species, in terms of prediction errors for overall populations or transects. Estimates of individual transects using low and slow sampling generally produced the lowest deviation from actual transect density. Often the best choice of flight plan comes down to a trade-off between covering more area (by flying faster or higher) or sampling with greater precision, and whether the resultant flight plan will dramatically affect population-level estimates. Depending on the magnitude (and likely the structure) of detection errors, the performance ranking of flight plans may completely reverse, as seen for 
"koala" movement (Figures 2d, 3d). Spatially structured spread of invasive ("weed" and "rust") species, and high fidelity detection along individual transect (perpendicular to the spread direction) suggests possible application to surveys for an advancing invasion front.

To gain these insights, we deliberately modelled three artificial species with widely contrasting spatial habits. As with any model, all components of our model could be made more realistic or include more subcomponents, such as exact image-resolution calculations or realistic empirical movement ecology. Calibrating the model to reflect actual species is desirable and may reveal further subtleties of UAV monitoring strategies. Parameterisation of the model with empirically derived detection errors would also be extremely useful. Further deconstruction of "detection" into components of image-capture, image analysis, and statistical estimation of population size, change or extent could yield insights into which components of surveillance need improvement. We could also incorporate reducing error over time, as identification algorithms are improved or uncertain identifications are crossed-checked with ground truthing.

We have nonetheless highlighted some occasions where the sampling regime may interact positively or negatively with species movement patterns across space, providing either opportunities or challenges for maximising the benefit of UAV technology to ecological monitoring. Our modelling approach could be applied to shed light on the utility of other automated surveillance devices (Jurdak et al. 2015) such as robotic cameras, and in the freshwater and marine realms.

\section{ACKNOWLEDGMENT}

We acknowledge the support of the Australian Government's Cooperative Research Centres Program.

\section{REFERENCES}

Anderson, K. and Gaston, K.J. (2013). Lightweight unmanned aerial vehicles will revolutionize spatial ecology. Frontiers in Ecology and the Environment, 11, 138-146.

Campbell, K.J., Beek, J., Eason, C.T., Glen, A.S., Godwin, J., Gould, F. et al. (2015). The next generation of rodent eradications: Innovative technologies and tools to improve species specificity and increase their feasibility on islands. Biological Conservation, 185, 47-58.

Carnegie, A. and Cooper, K. (2011). Emergency response to the incursion of an exotic myrtaceous rust in Australia. Australasian Plant Pathology, 40, 346-359.

Field, S.A., Tyre, A.J., Jonzen, N., Rhodes, J.R. and Possingham, H.P. (2004). Minimizing the cost of environmental management decisions by optimizing statistical thresholds. Ecology Letters, 7, 669-675.

Hodson, D.P. (2011). Shifting boundaries: challenges for rust monitoring. Euphytica, 179, 93-104.

Jorgensen, K., Cannon, R. and Muirhead, I. (2003). Guidelines for the Establishment of Pest Free Areas for Australian Quarantine. Report for Plant Health Australia Ltd and Australian Government Department of Agriculture, Fisheries and Forestry, 84pp.

Jurdak, R., Elfes, A., Kusy, B., Tews, A., Hu, W., Hernandez, E., Kottege, N. and P. Sikka (2015). Autonomous surveillance for biosecurity. Trends in Biotechnology, 33, 201-207.

Kalaris, T., Fieselmann, D., Magarey, R., Colunga-Garcia, M., Roda, A., Hardie, D. et al. (2014). The role of surveillance methods and technologies in plant biosecurity. In: The Handbook of Plant Biosecurity (eds. Gordh, F and McKirdy, S). Springer Dordrecht, Netherlands, pp. 309-337.

McDonald-Madden, E., Baxter, P.W.J. and Possingham, H.P. (2008). Subpopulation triage: How to allocate conservation effort among populations. Conservation Biology, 22, 656-665.

Sinden, J., Jones, R., Hester, S., Odom, D., Kalisch, C., James, R. and O. Cacho (2004). The economic impact of weeds in Australia. In: Technical Series. CRC for Australian Weed Management. 